\title{
Research and Application of Satellite Integration Design Based on MBD
}

\author{
Wu Ruilan, , Yang Liuying, Xia Yongquan, Jin Xiaokang, Jiang Hao \\ Institute of Telecommunication Satellite, China Academy of Space Technology, Beijing 100094, China
}

\begin{abstract}
As satellite integration design and implementation become more and more complex and difficult, a MBD model structure suit for satellite integration design is proposed in this paper, to ensure data consistency and the design work more efficiency. In order to help designers build normative MBD model efficient, a rapid MBD modeling tool has been developed and already been validated in several satellite development projects.
\end{abstract}

\section{General Instructions}

Satellite integration is the detailed design of mechanical aspects in satellite overall design. Integration implementation is the process that integration technologist and workers assemble the qualified onboard products into a complete satellite according to the integration design and technological requirements. ${ }^{[1]}$

With more tasks, shorter development cycle, while the mission is becoming more and more complex, satellite integration design and implementation is getting much more difficult than ever before. These challenges are meant to introduce a new way of satellite integration design.

Model-based definition (MBD) is an advanced digital definition methods that make all related design information structured inside native 3D models. A satellite integration design solution and implementation based on MBD was proposed in this paper, and a MBD modeling tool has been developed to help designers build MBD models of satellite products quickly and normatively.

\section{Satellite Integration Design}

Satellite integration design is the design process of planning and promoting demands on satellite AIT requirements, and finally writing them on documents and drawings, according to requirements from system, subsystem, and comprehensive test. ${ }^{[2]}$ Following is the concrete design content of satellite integration:

Mounting hole design of onboard product on deck, including the number, aperture size, depth, specification, and etc;

Equipment assemble design;

$>$ Cable route design and assemble design;

$>$ Pipeline of propulsion system route design and assemble design;

$>$ Grounding system design.

$>$ Onboard subordinate parts design.

Satellite integration design and implementation, take almost half of the development cycle, is an important link in satellite development process. The content mentioned above is complex and complicated, traditional design method and expression can hardly meet more and more satellite program research needs: 
$>$ Integration design datum are scattered in different documents and drawings, which is not good for later maintenance.

$>$ Easy to lead important information miss because integration technologists have to collect necessary information from several documents and drawings to compile process.

$>$ Rely on documents and drawings too much that the presentation sometime is not accurate and easy to form misunderstanding.

$>$ Low degree of automation that much data extraction are carried out manually which lead to low inefficient.

\section{MBD Solution}

3D models have been widely used in satellite integration design today, but 2D drawing is still used for detail description. Because of the existence of both 3D models and 2D drawings, designers can hardly ensure data from two sources consistency.

There are the advantages by adopting full 3D design and manufacturing mode without 2D drawing:

Ensure data consistency;

Reduce the possibility of mistakes by misunderstanding;

Shorten product development cycle;

> Promote concurrent engineering and product lifecycle management.

Full 3D design and manufacture is not simply to add information expressed in 2D drawing into 3D models, but to explore a much more efficient design information representation that easy to understand by making full use of 3D model advantages.

MBD technology provides a way to make satellite full 3D digital integration design reality. With MBD, most of the data related to the product can be stored in the $3 \mathrm{D}$ CAD model. At present, there is not a unique approach for MBD scenarios, this paper focus on establishing an approach suitable for satellite integration design and implementation. There are two types of satellite product often used :
Products only for satellite integration, such as the products delivered directly by the suppliers. During satellite integration design process, these products only need information of various interface like mechanical interface, thermal control interface, electrical interface, etc.

Products for manufacture and satellite integration, such as onboard subordinate parts. Those products need both manufacturing definitions about explicit dimensions, functional tolerances, and manufacturing annotations for manufacture, and information of various interfaces for satellite integration.

From above description, not only manufacturing definition, but information of mass property, mechanical interface, thermal control interface, and electrical interface should be embedded in 3D models. A MBD structure based on satellite integration design was established, just as Figure 1 shows.

Following is the detailed data added in native 3D model:

\section{Basic information}

- product designation;

- product code;

- supplier;

- Inheritance;

- Maturity;

- etc.

$\mathrm{MCI}($ mass, centroid, inertia) information

- mass;

- centroid location;

- moment of inertia.

Manufacturing oriented information

- functional tolerancing and annotations (FT\&A);

- manufacturing requirements.

Integration implementation oriented information

- Mechanical interface information

- Electrical interface information

- Thermal control interface information

Other information 
Present CAD technologies make manufacturing oriented information easily added in the $3 \mathrm{D}$ model by FT\&A module of 3D CAD software. However, integration implementation oriented information relies heavily on products' characteristics, production mode and process. There is no standard MBD model format and content for all the products. MBD model structure of satellite products should be customized based on their own characteristics.

\section{MTERICS}

In order to build normative MBD 3D models efficiently, METRICS( Mechanical Engineering Tri-dimensional Interface Control System) toolset was developed in CATIA environment. METRICS can help designers build the MBD model of an onboard product by writing all needed data into the native 3D model in document-driven form. The data written in is stored in geometric set form and exchanged while the model used. Figure 2 showed a typical MBD model structure built by METRICS.

Following is the data written in the MBD models by METRICS:

$>$ Product basic information: such as product designation, boxcode, supplier,

MCI(Mass, centroid, inertia ) information,

$>$ Mechanical mounting information;

- Mechanical grounding or insulation information;

Basic thermal performance information;

Dlectrical connector information;

RF Ports definition and identification;

$>$ Special announcement or description of the product.

Once satellite configuration and layout are completed, integration designer have to extract and summarize related information from product MBD models as soon as possible, then submit to structural designer or satellite integration technologist. Therefore, associated tools are developed:

METRICS_AIT is used to extract mechanical mounting information from all models in structured tree.

METRICS MCI is used to extract MCI information from models in the local coordinate system, then convert to satellite mechanical coordinate system and do summary calculate.

> METRICS_Drilling_Map is used to extract mounting hole information from models in local coordinate system, then convert to satellite mechanical coordinate system and do summary calculate.

METRICS toolset has been validated in several satellite development projects and is constantly improved perfect according to the application. More data beside above mentioned can be involved in, to make MBD model more complete, not only used in satellite integration design, but satellite concurrent engineering and lifecycle management.

\section{Conclusion}

A MBD model structure of satellite products was proposed in this paper, which contained most onboard products' integration and implementation information. The MBD model used in satellite design made satellite lifecycle 3D digital design, manufacture and management reality.

A rapid MBD modeling toolset METRICS was developed to help integration designers build normative MBD 3D models efficiently and had been validated efficiently and accurately in several satellite programs.

In order to provide better service for satellite concurrent engineering and lifecycle management, more data beside this paper mentioned can be involved in the MBD model structure for the better application. It can be predicted that MBD is carrying more information for design, simulation and implementation to form an actual virtual prototype. Then TBD technology will be developed into MBSE technology.

\section{Acknowledgements}

This work has been supported by Project of Research and Application of Knowledge Management Technology in Aerospace Major Equipment Innovative Development Engineering under the Project Number 2015BAF18B01. 


\section{Reference}

[1] M. Alemanni, F. Destefanis, E. Vezzetti. Model-based definition design in the product lifecycle management scenario[J]. International Journal of Advanced Manufacturing Technology, 2011(1/4): 1-14.

[2] Leilie Yin, Dunbing Tang, Qi Wang, Inayat Ulah, Haitao Zhang. Engineering Change Management of Product Design Using Model-Based Definition Technology[J].Journal of Computing and Information Science in Engineering, 2016,17(4): 041006-041006-19

[3] Wenhua Zhu, Matthieu Bricogne, Alexandre Durupt, Sebastien Remy, Baorui Li, Benoit Eyanard. Implementations of Model Based Definition and Product Lifecycle Management Technologies: a Case Study in Chinese Aeronautical Industry[J].
IFAC-Papers On Line,2016, 49(12):485-490

[4] $\mathrm{Xu}$ Fuxiang. Introduction to Satellite Engineering $[\mathrm{M}]$. Beijing, China Astronautic Publishing House, 2003.

[5] Chen Xiangdong, Zhang Wangjun, Pan Yanhua. Study on Spacecraft Digital Integration Design[j]. Spacecraft Engineering, 2008, 17(6):64-67.

[6] Feng Tongneng, Wang Zhenyang, Mengjinhui. Application and Development of MBD in Digital Collaborate Manufacturing [J]. Journal of Nanjing University of Aeronautics \& Astronautics, 2012,44:132-137.

[7] Chandrasegaran S.K., Ramani K., Sriram R.D., Horváth I., Bernard A., Harik R.F.. The Evolution, Challenges, and Future of Knowledge Representation in Product Design Systems[J]. Computer-Aided Design, 2013， 45(2): 204-228.

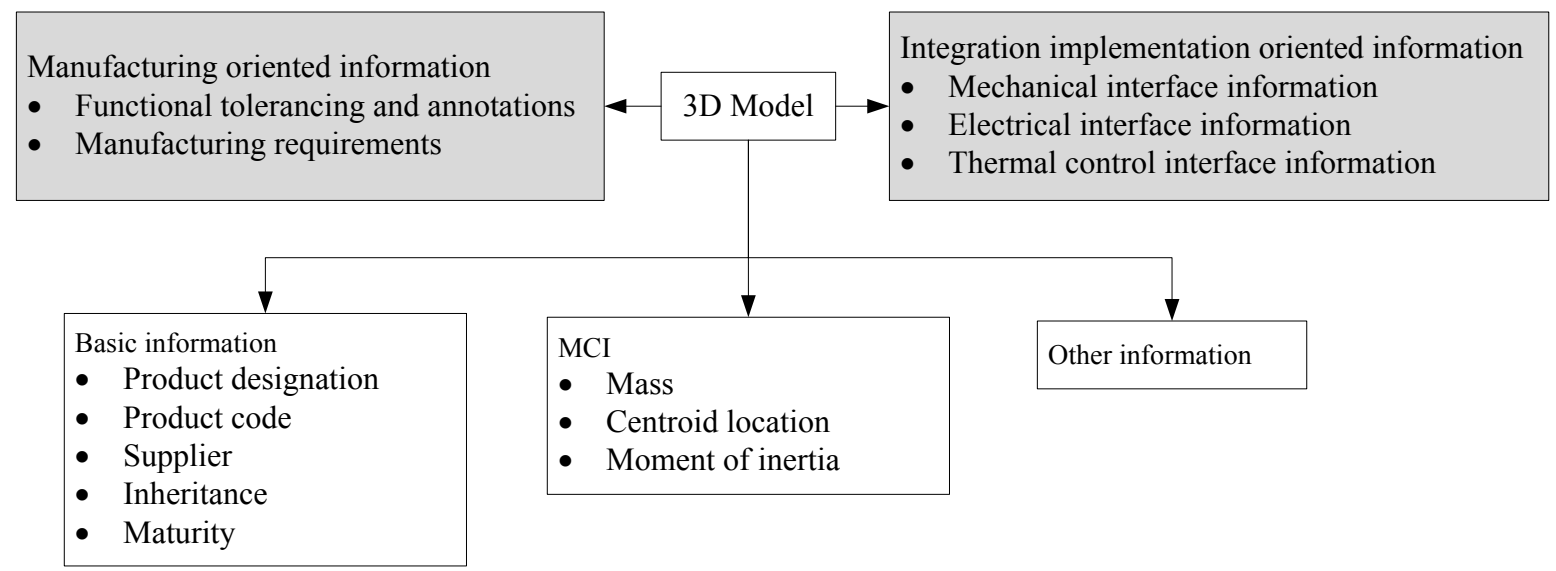

Fig.1 MBD Structure based on satellite integration design

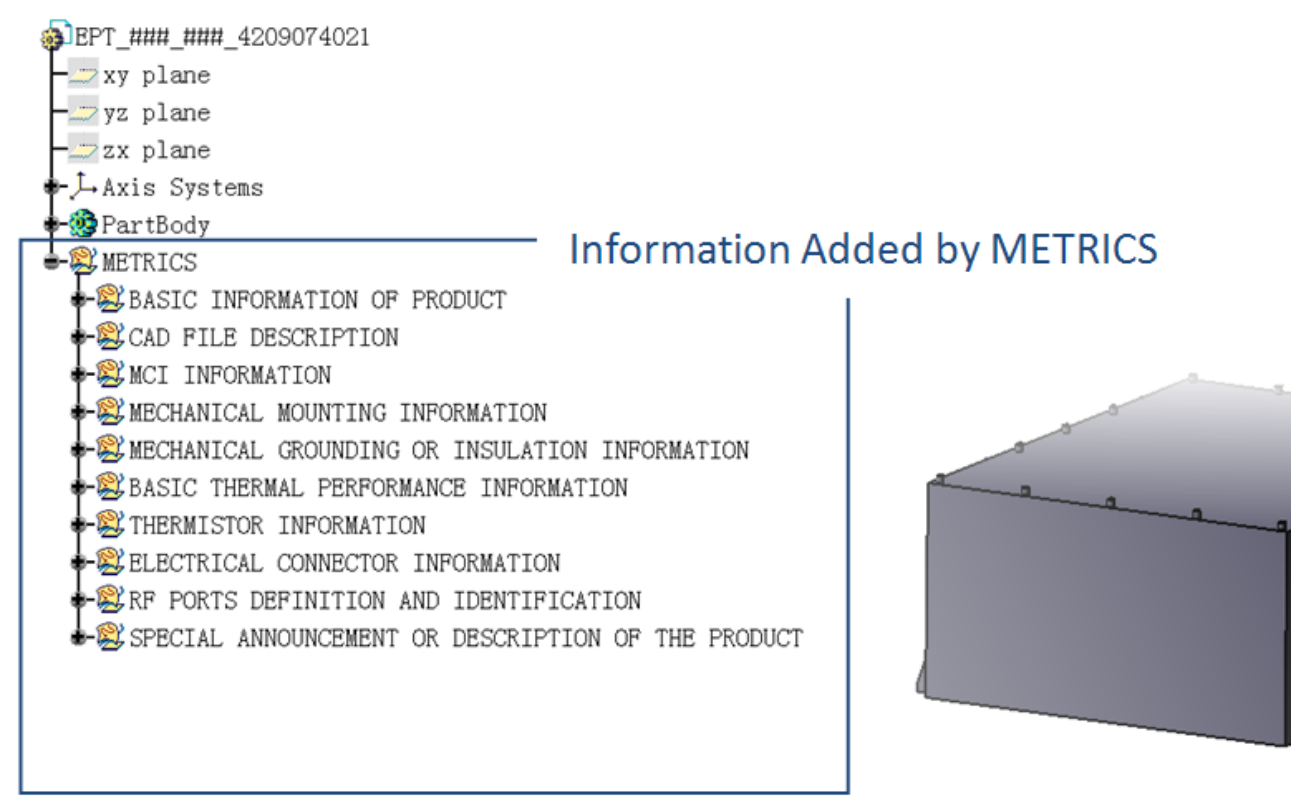

Fig.2 A typical MBD model structure built by METRICS 